\title{
Geology and Water Quality at Selected Locations in the San Antonio Area Texas, Progress Report, 1969
}

Edwards Underground Water District San Antonio, Texas

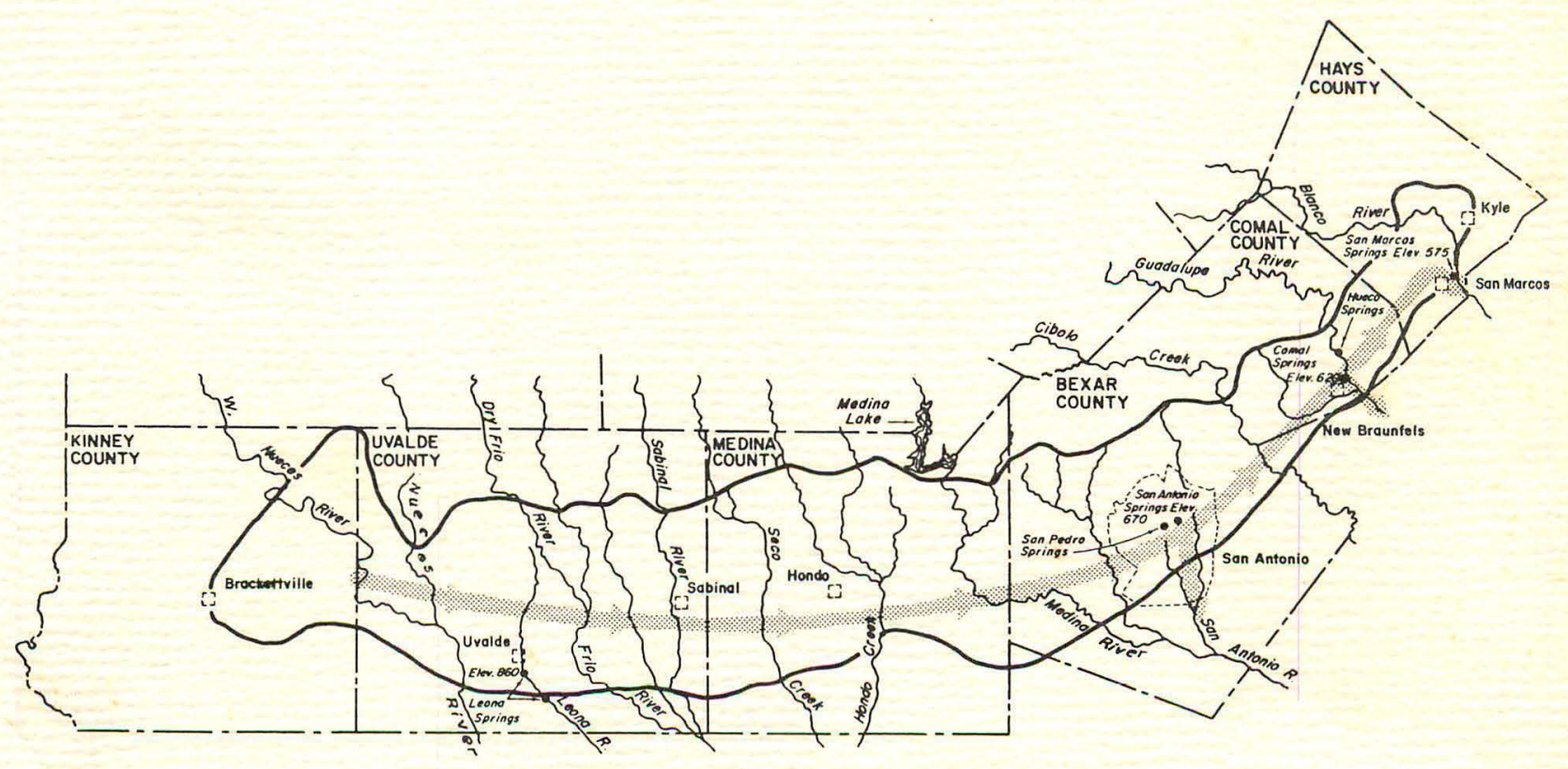

Prepared in cooperation with the U.S. Geological Survey and the Texas Water Development Board 


\section{EDWARDS UNDERGROUND WATER DISTRICT}

\section{Tower Life Building}

San Antonio, Texas 78205

GEOLOGY AND WATER QUALITY AT SELECTED LOCATIONS

IN THE SAN ANTONIO AREA, TEXAS

PROGRESS REPORT, 1969

\section{By}

R. D. Reeves and J. F. Blakey United States Geological Survey

Prepared by the U.S. Geological Survey in cooperation with the Edwards Underground Water District and the Texas Water Development Board 


\section{CONTENTS}

Page

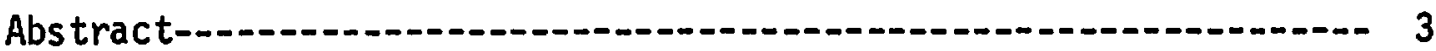

Introduction-1.-1

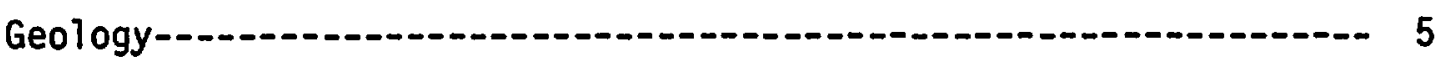

Water quality-1.-1 8

Continuing studies-1

References cited-1.-1 12

\section{ILLUSTRATION}

Figure 1. Map showing water-quality data-collection sites in the San Antonio area and geology of part of

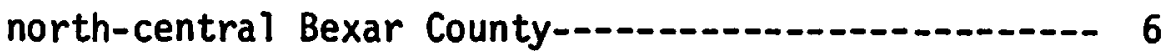

\section{TABLES}

Table 1. Quality-of-water data from wells and springs in the San Antonio area--.-.- 13

2. Quality-of-water data from sites other than wells and springs in the San Antonio area--.......-.- 16 


\title{
GEOLOGY AND WATER QUALITY AT SELECTED LOCATIONS \\ IN THE SAN ANTONIO AREA, TEXAS \\ PROGRESS REPORT, 1969 \\ By \\ R. D. Reeves and J. F. Blakey \\ United States Geological Survey
}

\begin{abstract}
The Edwards aquifer is the principal source of water supply for the San Antonio area. Increasing urban development on or adjacent to the recharge area of the aquifer is causing great concern because of possible pollution of the ground water. A detailed map of the surface geology has been prepared for areas where the greatest threat of pollution exists. Water-quality data are being collected throughout the San Antonio area to provide background reference information and to detect any current pollution of the ground water in the Edwards and associated limestones.

Mapping of the surface geology will continue in areas where waste waters may be recharging the aquifer. Water-quality data will be collected at additional sites, and some sites will be resampled where pollution may be occurring or where more background data are needed.
\end{abstract}




\section{INTRODUCTION}

Geologic and hydrologic investigations of the Edwards and associated limestones (aquifer) in the San Antonio area have been carried on for many years by the U.S. Geological Survey in cooperation with the Texas Water Development Board and local agencies. In recent years, the studies in cooperation with the Edwards Underground Water District have been chiefly hydrologic. However, since 1968, local water users have become greatly concerned about the possible pollution of ground water in the Edwards aquifer resulting from urban development on or adjacent to the recharge area.

The Edwards aquifer is the principal source of water for municipal supply, defense establishments, industrial use, and agricultural use. One of the main sources of potential pollution is the infiltration of effluent from septic tanks and from sewage-treatment plants that are in the recharge area or that discharge their effluent into dry stream beds that cross the recharge area. Other possible sources of pollution are sanitary landfills, industrial wastes, surface runoff from residential subdivisions, and leakage from sewage collection systems. 
During 1968-69, field work consisted of detailed mapping of the surface geology, with special emphasis on those areas in north-central Bexar County where sewage-treatment plants are either operational or planned, and the collection of water-quality data for selected wells and streams in the San Antonio area (fig. 1). Water samples were collected below sewage plants at the request of local agencies. Quality data for the waste waters are an important part of the study, particularly the determination of minor-element concentrations. Minor elements identified in the waste water may serve as tracers for determining movement of the waste water in the aquifer. Chemical and biological analyses of the water samples collected during the investigation will be used as historical reference data to determine changes in water quality and to detect any current pollution of the ground water in the Edwards and associated limestones.

\section{GEOLOGY}

The geologic units exposed in the vicinity of the sewage plants are, from oldest to youngest: The Edwards and associated limestones, Grayson Shale, Buda Limestone, Austin Chalk, Anacacho Limestone, and Quaternary alluvium (fig. 1). Except for the alluvium, the formations dip generally toward the southeast at an angle steeper than the slope of the land surface; thus, the older formations crop out in the northwest part of the area. In some places the continuity of the formations is disrupted by faulting. 
In part of the area, the Edwards and associated limestones are overlain by formations, such as the Grayson Shale and the Anacacho Limestone, that for all practical purposes are impervious to the infiltration of pollutants. It seems probable, therefore, that the effluent from the airport sewage plant or the proposed sewage plant near Wetmore would not percolate downward to the aquifer.

Seepage investigations during periods of flood show that in the vicinity of the San Pedro Hills sewage plant, some of the flow is lost to the Austin Chalk. Because the Austin Chalk is in contact with the Edwards and associated limestones as a result of faulting, it seems highly likely that the effluent from the plant will eventually make its way into the Edwards aquifer.

The greatest threat of pollution exists where pollutants are deposited on or flow into the fractured and cavernous outcrop of the Edwards and associated limestones. In general, the greatest concentrations of solution-enlarged fractures are found in the streambeds (fig. 1), which are dry except during periods of high rainfall and flood runoff. 


\section{WATER QUALITY}

During this study, waters of the San Antonio area have been analyzed for a number of properties and characteristics--including inorganic parameters, organic and biological parameters (nutrients, bacteria, and biochemical-oxygen demand), selected pesticides, and minor elements. Although sampling has been concentrated in the areas north and northwest of San Antonio, samples have been collected elsewhere in the San Antonio area, including one or more sites in seven counties (fig. 1).

A11 water-quality data collected through August 1969, except pesticide data, are shown in tables 1 and 2. Waters from the following sites (fig. 1) were analyzed for pesticides ( 9 insecticides and 3 herbicides) during the study, and all concentrations were less than $0.005 \mu \mathrm{g} / \mathrm{l}$ (micrograms per liter):

$$
\begin{aligned}
& \text { AY-68-28-903 } \\
& \text { AY-68-29-204 } \\
& \text { AY-68-29-403 } \\
& \text { AY-68-29-810 }
\end{aligned}
$$$$
\text { AY }-68-36-410
$$$$
\text { JJ-55-63-701 }
$$$$
\text { JJ-55-63-703 }
$$$$
\text { Site } 6 \text { - Frio River at Concan. }
$$ 
A water sample collected downstream from the airport sewage plant (site C) in September 1968 contained $0.15 \mu \mathrm{g} / 1$ DDD [1, 1-dichloro-2, 2-bis (p-chloropheny1) ethane]; $0.10 \mu \mathrm{g} / 1$ DDE [1, 1-dichloro-2, 2-bis (p-chlorophenyl) ethylene $] ; 0.06 \mu \mathrm{g} / 1$ DDT $[1,1,1$-trichloro-2, 2-bis (p-chloropheny 1 ) ethane]; and $0.02 \mu \mathrm{g} / 1$ Dieldrin. Other insecticides and herbicides were less than $0.005 \mu \mathrm{g} / 1$. Concentrations of pesticides permissible in public water supplies are given in the following table:

(Adapted from Water Quality Criteria--Report of the National Technical Advisory Committee to the Secretary of the Interior: Federal Water Pollution Control Administration, 1968)

\begin{tabular}{|c|c|c|}
\hline \multicolumn{2}{|c|}{$\begin{array}{c}\text { Insecticide } \\
\text { (micrograms/liter) }\end{array}$} & $\begin{array}{c}\text { Herbicide } \\
\text { (micrograms/liter) }\end{array}$ \\
\hline Aldrin & 17 & $2,4-D$ plus \\
\hline DDT & 42 & $2,4,5-T$ plus \\
\hline Dieldrin & 17 & Silvex \\
\hline Endrin & 1 & \\
\hline Heptachlor & 18 & \\
\hline Heptachlor epoxide & 18 & \\
\hline Lindane & 56 & \\
\hline
\end{tabular}


Analyses of water for inorganic parameters and minor elements, in addition to portraying existing conditions, provide background data for determining movement and volume of future recharge that may have significant quantities of one or more of these minerals--a possible source of pollution may provide its own tracer.

Nutrients (nitrogen and phosphorus compounds) and BOD (biochemical oxygen demand) are indicators of pollution. However, the sanitary condition of water supplies is routinely based on an evaluation of the concentrations of coliform bacteria. The use of coliform organism as an indicator of pollution is discussed by the U.S. Public Health Service (1962, p. 14). In summary, the report states that the presence of any type of coliform organism is undesirable in drinking water. The presence of fecal coliform organisms indicates recent pollution, whereas the presence of organisms determined as total coliforms includes bacteria with longer survival times in water and may indicate less recent pollution.

Fecal streptococci are also indicators of fecal pollution, and together with fecal coliforms, they can provide information on the source of pollution. For example, Geldreich (1966, p. 102) reported an average ratio of fecal coliforms to fecal streptococci of 4.4 in man, 0.04 in pigs, and less than 0.1 in other domestic animals. 
The short survival time and the possible increase in population of bacteria in water make it imperative that analysis for these organisms be started as soon as possible after a sample is collected. Portable equipment is being used in this study and determinations are begun at the sampling site. Such equipment for determining fecal coliforms and fecal streptococci was not available until 1969.

\section{CONTINUING STUDIES}

Mapping of surface geology will continue, with special emphasis on those areas where waste waters may be recharging the aquifer. Waterquality data will be collected at additional sites, and some sites will be resampled for determination of selected parameters. Additional pesticide data will be collected in areas of possible pollution and from selected wells to provide background data for the study area. Bacteria counts and nutrient concentration will be determined at all new and resampled sites. 


\section{REFERENCES CITED}

Geldreich, E. E., 1966, Sanitary significance of fecal coliforms in the environment: Federal Water Pollution Control Adm. Pub. WP-20-3, p. 122.

National Technical Advisory Committee to the Secretary of the Interior, 1968, Water Quality Criteria: Federal Water Pollution Control Adm., p. 20-83.

U.S. Public Health Service, 1962, Public Health Service drinking water standards: U.S. Public Health Service Pub. 956, 67 p., 1 fig. 
Table 1.--Quality-of-pater data fron welly and uprings in the San Antonlo area

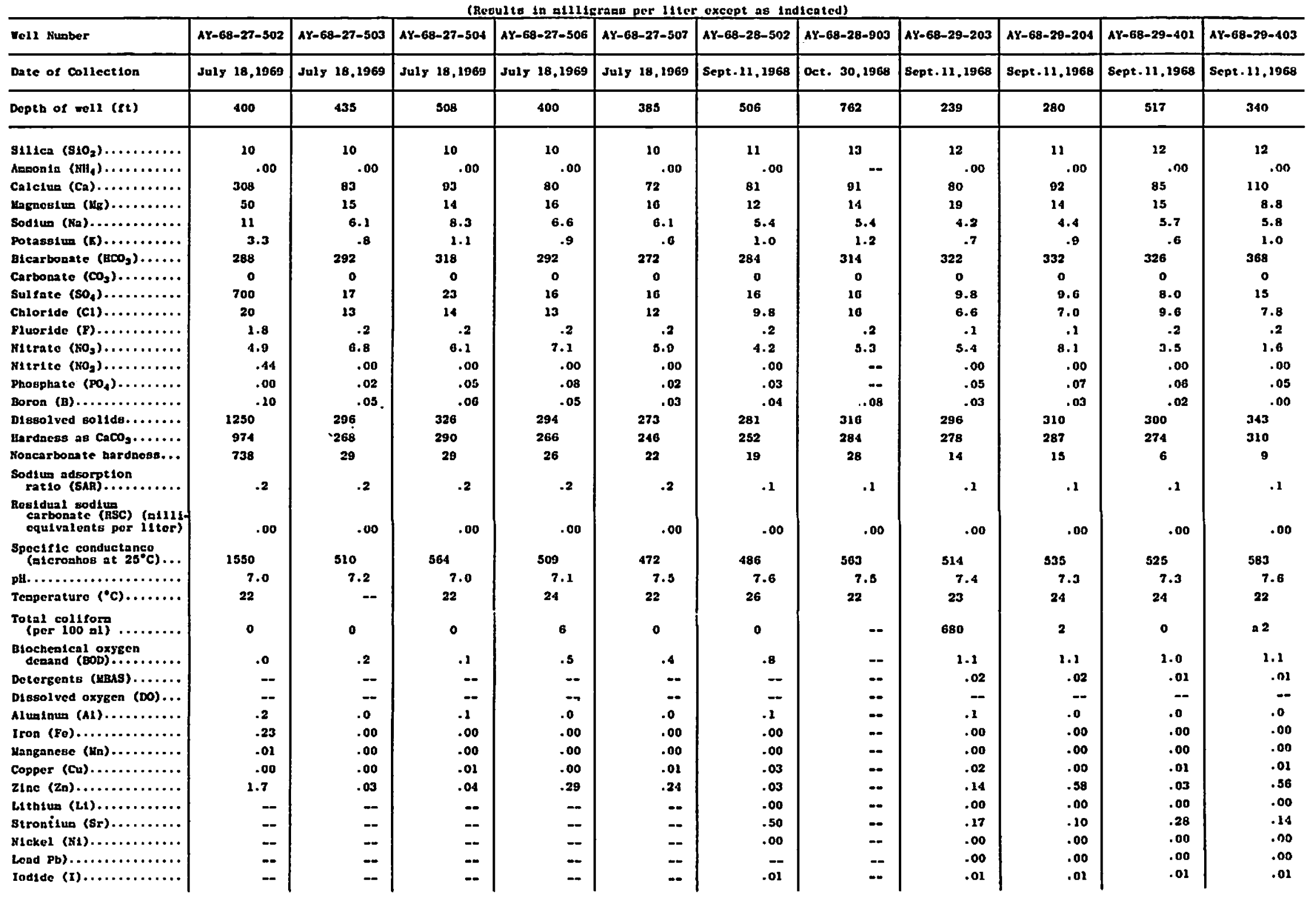


Table 1.--Quality-of-mter data fron gel1s and springe in the San Antonlo area--continued

(Results in alligrans per liter except an indicatod)

\begin{tabular}{|c|c|c|c|c|c|c|c|c|c|c|c|}
\hline \multicolumn{12}{|c|}{ (Results in all11grans per liter except an indicatod) } \\
\hline Tell Number & $A Y-68-29-405$ & AY-68-29-407 & $A Y-68-29-408$ & AY-68-29-409 & AY-68-29-501 & AY-68-29-502 & AY- $68-29-503$ & AY-68-29-809 & $A Y-68-29-809$ & AY-68-29-810 & AY-68-35-307 \\
\hline Date of collection & Sept.11,1068 & Sopt.11,1068 & Sept. 11, 1968 & Apr. 2,1969 & Jan. 11,1960 & Sept.11.1068 & Sept.11,1068 & Oct. 31,1968 & Apr. 2, 1969 & Apr. 2, 1969 & July 18.1969 \\
\hline Depth of vell (ft) & 395 & 340 & 390 & 460 & $350 \pm$ & 264 & 349 & 364 & 364 & 300 & 291 \\
\hline silice $\left(\mathrm{SiO}_{2}\right) \ldots \ldots$ & 12 & 12 & 12 & 13 & 14 & 12 & 11 & 9.3 & 13 & 12 & 14 \\
\hline Amonla $\left(\mathrm{s}_{1}\right), \ldots \ldots \ldots$ & .00 & .00 & .00 & -- & .00 & .00 & .00 & .00 & & & .00 \\
\hline Caletua $\left(C_{a}\right) \ldots \ldots \ldots \ldots$ & 80 & 09 & 100 & 98 & 108 & 98 & 90 & 74 & $\mathbf{7 7}$ & 88 & 81 \\
\hline Hagnosiun $\left(H_{G}\right) \ldots \ldots \ldots \ldots$ & 11 & 8.4 & 21 & 12 & 11 & 12 & 11 & 15 & 15 & 15 & $\mathbf{3 1}$ \\
\hline Sodtue $(\mathrm{k} a) \ldots \ldots \ldots \ldots \ldots$ & 8.6 & 10 & 5.6 & 2.5 & 6.2 & 5.4 . & 4.4 & 13 & 9.7 & 6.9 & 16 \\
\hline Potassiun $(k) \ldots \ldots \ldots \ldots$ & 1.2 & .0 & -- & -- & -- & 1.0 & .7 & & -- & & 3.0 \\
\hline Bicarbonate $\left(\mathrm{HCO}_{3}\right) . . . .$. & 308 & 324 & 344 & 344 & 372 & 350 & 320 & 284 & 276 & 300 & 348 \\
\hline Carbonate $\left(\mathrm{CO}_{3}\right) \ldots \ldots \ldots$ & o & $\mathbf{0}$ & 0 & o & o & 0 & $\mathbf{0}$ & o & 0 & o & 0 \\
\hline sulfate $\left(\mathrm{so}_{4}\right) \ldots \ldots \ldots \ldots$ & 13 & 27 & 7.2 & 6.8 & 6.8 & 7.2 & 4.8 & 15 & 23 & 23 & 33 \\
\hline Cbloride (C1) ............ & 12 & 14 & 8.0 & 6.5 & 7.8 & 8.6 & 6.6 & 18 & 16 & 11 & 32 \\
\hline Fluoride $(g), \ldots \ldots \ldots \ldots$ & .1 & 1.3 & .1 & .1 & .1 & .1 & .1 & .3 & .2 & .2 & 1.0 \\
\hline vitrate $\left(N_{0}\right), \ldots \ldots \ldots \ldots$ & 8.0 & 2.0 & 3.6 & 1.4 & 5.8 & 3.0 & 3.5 & .0 & 1.8 & 6.0 & .0 \\
\hline Mitrite $\left(\mathrm{No}_{2}\right) \ldots \ldots \ldots \ldots$ & .00 & .00 & .00 & .01 & .00 & .00 & .00 & .00 & .09 & .01 & .00 \\
\hline phosphate $\left(\mathrm{PO}_{4}\right) \ldots \ldots \ldots \ldots$ & .09 & .05 & .05 & .04 & .08 & .03 & .03 & .03 & .03 & .04 & .02 \\
\hline Boron (B) $\ldots \ldots \ldots \ldots \ldots \ldots$ & .03 & .04 & .04 & .03 & .22 & .03 & .02 & .04 & .05 & .04 & .00 \\
\hline Dissol ved solids......... & 304 & 324 & 318 & 309 & 343 & 320 & 280 & 285 & 292 & 308 & 384 \\
\hline Hardsess $28 \mathrm{CaCO}_{3} \ldots \ldots$ & 270 & 282 & 294 & 294 & 314 & 204 & 270 & 246 & 254 & 276 & 330 \\
\hline Noncarbonate mardncess... & 17 & 16 & 12 & 12 & 10 & 7 & $\mathbf{8}$ & 14 & 28 & 30 & 14 \\
\hline $\begin{array}{l}\text { Sodium } \\
\text { rat10 } \\
\text { adsorption } \\
\text { (SAR)............... }\end{array}$ & .2 & .3 & .1 & .1 & .1 & .1 & .2 & .4 & .3 & .2 & .4 \\
\hline $\begin{array}{l}\text { Residual sodiun } \\
\text { carbonate (RSC) (nIII1- } \\
\text { equilvalents per ilter) }\end{array}$ & .00 & .00 & .00 & .00 & .00 & .00 & .00 & .00 & .00 & .00 & .00 \\
\hline $\begin{array}{l}\text { Spectife conductance } \\
\left.\text { (alcronhos at } 25^{\circ} \mathrm{C}\right) . .\end{array}$ & 572 & 348 & 546 & 547 & 393 & 559 & 531 & 517 & 311 & 320 & 659 \\
\hline pi............................. & 7.6 & 7.5 & 7.9 & 7.2 & 6.9 & 7.7 & 7.6 & 7.0 & 7.4 & 7.3 & 7.2 \\
\hline Texperature $\left({ }^{\circ} \mathrm{C}\right) . . . \ldots$. & 24 & 22 & 23 & 22 & 22 & 22 & 24 & -- & 23 & 23 & 26 \\
\hline $\begin{array}{l}\text { Totn1 colleor } \\
\text { (per } 100 \mathrm{al}) \ldots \ldots \ldots\end{array}$ & 0 & & 1 & & & 0 & 8400 & 0 & $-\infty$ & 0 & 0 \\
\hline $\begin{array}{l}\text { Blochenical oxygen } \\
\text { denand (BOD) } \ldots \ldots \ldots \ldots\end{array}$ & 1.8 & 1.1 & 1.3 & .3 & .3 & 2.1 & 1.5 & 3.0 & .6 & .3 & .3 \\
\hline Detergents (MBAS)....... & .02 & .03 & .02 & .10 & - & .04 & .00 & .04 & .16 & .03 & - \\
\hline Dissolved oxygen (DO)... & -- & -- & -- & -- & -- & -- & - & -- & -- & -- & - \\
\hline Aluninum (A1) $\ldots \ldots \ldots \ldots$ & .0 & .1 & .0 & - & .0 & .0 & .0 & .0 & -- & -- & .1 \\
\hline tron (Fe) $\ldots \ldots \ldots \ldots \ldots$ & .00 & .00 & .00 & .01 & .01 & .03 & .00 & 2.9 & .46 & .00 & 1.0 \\
\hline 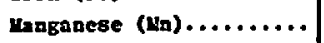 & .00 & .00 & .00 & .00 & .01 & .00 & .00 & .08 & .00 & .00 & .10 \\
\hline Copper (Cu) $\ldots \ldots \ldots \ldots \ldots$ & .01 & .02 & .00 & - & .40 & .01 & .01 & .01 & -- & -- & .00 \\
\hline $\operatorname{zinc}\left(z_{n}\right) \ldots \ldots \ldots \ldots \ldots$ & .07 & .08 & .23 & -. & .00 & .68 & .12 & .06 & -- & -- & .08 \\
\hline Litbiue (Li) $\ldots \ldots \ldots \ldots$ & .01 & .01 & .00 & - & .00 & .00 & .00 & .00 & -- & -- & - \\
\hline Stronftue $(\mathrm{S} x) \ldots . . . \ldots$ & .21 & .20 & .12 & -- & .14 & .14 & .10 & .43 & - & - & - \\
\hline Nickel (ki).$\ldots \ldots \ldots \ldots$ & .00 & .00 & .00 & -- & .00 & .00 & .00 & .01 & -- & -- & - \\
\hline Lead $(P b), \ldots \ldots \ldots \ldots \ldots$ & .00 & .00 & .00 & -- & .00 & .00 & .00 & .00 & -- & -- & -- \\
\hline Iodide (1).............. & .01 & .01 & .01 & - & .02 & .01 & .01 & .04 & -- & -- & -- \\
\hline
\end{tabular}


Table 1.--Quallty-of-water data fron sells and springy in the San Antonto area--continued

(Results in ailligrane per liter except ae indicated)

\begin{tabular}{|c|c|c|c|c|c|c|c|c|c|}
\hline Well Nuaber & $A Y-68-36-410$ & DX-68-15-901 & DX-68-16-802 & DX-68-23-201 & JJ-55-63-701 & JJ-55-63-703 & 2R-67-01-701 & $2 R-67-01-801$ & 2L-67-09-101 \\
\hline Date of Collection & Jan. 11,1969 & Oct. 31,1968 & Jan. 11,1060 & Jan. 11,1969 & Nov. 7, 1968 & Nov. 7,1968 & oct. 31,1968 & act. 31,1968 & oct. 31,1968 \\
\hline Depth of well (ft) & 604 & -- & 190 & 367 & 563 & 346 & -- & -- & 229 \\
\hline silica $\left(s_{10}\right) \ldots \ldots \ldots \ldots$ & 13 & 11 & 12 & 12 & 12 & 14 & 11 & 11 & 12 \\
\hline AEsonia $\left(N_{4}\right) \ldots \ldots \ldots$ & .00 & .00 & .00 & .00 & -- & -- & .00 & .00 & .00 \\
\hline Calciug (Ca)............ & 68 & 88 & 82 & 91 & 46 & 72 & 92 & 82 & 100 \\
\hline Magneglus $\left(\mathrm{Hg}_{\mathrm{g}}\right) \ldots \ldots \ldots$ & 15 & 15 & 16 & 7.6 & 26 & 4.8 & 12 & 19 & 16 \\
\hline Sodius $\left(M_{a}\right) \ldots \ldots \ldots \ldots$ & 9.2 & 7.4 & 12 & 11 & 12 & 15 & 14 & 7.4 & 23 \\
\hline Potaseiva (K).......... & -- & -- & -- & -- & & & -- & -- & -- \\
\hline alcarbonate $\left(\mathrm{HCO}_{3}\right) \ldots \ldots$ & 252 & 340 & 304 & 288 & 212 & 220 & 308 & 300 & 342 \\
\hline Carbonate $\left(\infty_{1}\right) \ldots \ldots \ldots$ & $\bullet$ & 0 & 0 & 0 & 0 & 0 & 0 & o & o \\
\hline sulfate $\left(s_{0},{ }_{1} \ldots \ldots \ldots \ldots\right.$ & 23 & 15 & 20 & 11 & 7.0 & 11 & 9.2 & 23 & 36 \\
\hline chloride (c1)........... & 11 & 14 & 34 & 16 & 16 & 22 & 13 & 19 & 23 \\
\hline Fluorlde $(p) \ldots \ldots \ldots$ & .1 & .3 & .1 & .1 & .4 & .2 & .2 & .3 & .3 \\
\hline Mitrate $\left(N_{0},{ }^{\prime}, \ldots \ldots \ldots \ldots\right.$ & 5.6 & 9.2 & 8.1 & 15 & 3.2 & 11 & 1.1 & .6 & 11 \\
\hline Nitrite $\left(\mathrm{NO}_{2}\right) \ldots \ldots \ldots$ & .00 & .04 & .00 & .00 & .00 & .00 & .00 & .00 & .07 \\
\hline Phosphate $\left(P_{4}\right) \ldots \ldots \ldots$ & .02 & .05 & .03 & .03 & -- & -- & .07 & .04 & .05 \\
\hline Boron (B) $\ldots \ldots \ldots \ldots \ldots \ldots$ & .05 & .04 & .07 & .00 & .04 & .07 & .01 & .01 & .05 \\
\hline DLseol ved solida......... & & 337 & 313 & 306 & 217 & 238 & 291 & 310 & 393 \\
\hline Haraness as $\mathrm{CaCO}_{3} \ldots \ldots$ & 231 & 306 & 270 & 258 & 181 & 199 & 279 & 282 & 316 \\
\hline Soncarbodate marcheso... & 24 & 28 & 22 & 22 & 7 & 19 & 26 & 36 & 33 \\
\hline 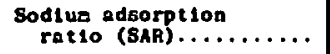 & .3 & .2 & .3 & .3 & .4 & .5 & .0 & .2 & .6 \\
\hline $\begin{array}{l}\text { Resldual sodiug } \\
\text { carbonate (RSC) (nt111 } \\
\text { equivalenta per liter) }\end{array}$ & .00 & .00 & .00 & .00 & .00 & .00 & .00 & .00 & .00 \\
\hline $\begin{array}{l}\text { Speciric conductance } \\
\left.\text { (a1 cronhos at } 25^{\circ} \mathrm{C}\right) . .\end{array}$ & 466 & 396 & 540 & $\$ 14$ & 393 & 455 & 534 & 574 & 658 \\
\hline p & 3.4 & 6.9 & 7.3 & 7.1 & 7.7 & 2.5 & 6.9 & 7.0 & 6.9 \\
\hline Teaperaturo $\left({ }^{\circ} \mathrm{C}\right) \ldots \ldots$ & 24 & -- & 23 & 23 & 23 & 18 & -- & - & -- \\
\hline $\begin{array}{l}\text { Total colltom } \\
\text { (per } 100 \mathrm{al}) \ldots \ldots\end{array}$ & 12 & 24 & o & $\mathbf{0}$ & $\mathbf{0}$ & 3100 & $\mathbf{0}$ & 8 & o \\
\hline $\begin{array}{l}\text { Biocherical oxysen } \\
\text { denand }(B O D) . . . . . . . . .\end{array}$ & .1 & .2 & .1 & .1 & .1 & .1 & .3 & .4 & .3 \\
\hline Detergents (kaus)....... & - & .00 & - & -- & - & - & .01 & .01 & .00 \\
\hline Dtseolved oxysen $(D O) \ldots$ & -- & -- & -- & -- & -- & -- & -- & -- & -- \\
\hline Aluginus $(A 1) \ldots \ldots \ldots$ & .0 & .0 & .0 & .0 & .1 & .0 & .0 & .0 & .1 \\
\hline Iron $\left(P_{0}\right) \ldots \ldots \ldots \ldots$ & .00 & .03 & .00 & .00 & .03 & .03 & .03 & .00 & .01 \\
\hline Uanganege $\left(U_{n}\right) \ldots \ldots \ldots$ & .00 & .00 & .00 & .00 & .00 & .00 & .02 & .00 & .00 \\
\hline Copper (Cu) $\ldots \ldots \ldots \ldots \ldots$ & .00 & .00 & .00 & .01 & .00 & .04 & .02 & .00 & .00 \\
\hline $\operatorname{zinc}\left(z_{n}\right) \ldots \ldots \ldots \ldots$ & .09 & .02 & .28 & 1.0 & .01 & .01 & .05 & .02 & .10 \\
\hline Lithlue (L1) $\ldots \ldots \ldots \ldots$ & .00 & .00 & .00 & .00 & .00 & .00 & .00 & .00 & .00 \\
\hline Strontiue (Sr)......... & .40 & .37 & .57 & .18 & .42 & .13 & .17 & .37 & .39 \\
\hline sickel $\left(k_{1}\right) \ldots \ldots \ldots \ldots$ & .00 & .01 & .00 & .00 & .00 & .00 & .00 & .00 & .00 \\
\hline $\operatorname{coad}(P b) \ldots \ldots \ldots \ldots \ldots$ & .00 & .00 & .00 & .01 & .00 & .00 & .00 &.$\infty 0$ & .00 \\
\hline sodide $(1) \ldots \ldots \ldots \ldots$ & .01 & .01 & .01 & .01 & .02 & .02 & .01 & .01 & .01 \\
\hline
\end{tabular}

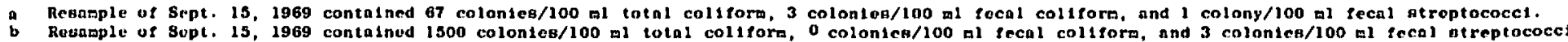


Tablo 2.--quallty-or-mater data trop altes other than wolls and opringe la tho San Antonto aroa

\begin{tabular}{|c|c|c|c|c|c|c|c|c|c|c|}
\hline & 81te 2 & site 2 & Site 3 & Site 3 & site 4 & site 4 & Site 3 & site 5 & Site 6 & S1te 6 \\
\hline & $\begin{array}{l}\text { Roul Prio A. } \\
\text { lalle above } \\
\text { nouth at } \\
\text { Loaky. }\end{array}$ & $\begin{array}{l}\text { East Prio R. } \\
\text { 1 alle above } \\
\text { gouth at } \\
\text { Loaky. }\end{array}$ & $\begin{array}{l}\text { Prio R. Rt } \\
\text { Hwy. 1120 } \\
\text { l gile below } \\
\text { Leaky. }\end{array}$ & 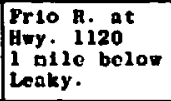 & $\begin{array}{l}\text { Prio R. at } \\
\text { Hery. 10so } \\
\text { above Garner } \\
\text { Park. }\end{array}$ & $\begin{array}{l}\text { Fr10 R. at } \\
\text { Hury. 10so } \\
\text { sbove Ga rnor } \\
\text { Park. }\end{array}$ & $\begin{array}{l}\text { Prio R. at } \\
\text { Hager's cross- } \\
\text { 1ng belö } \\
\text { Garnor Park. }\end{array}$ & $\begin{array}{l}\text { Frio R. at } \\
\text { Unger's cross- } \\
\text { 1ng boloz } \\
\text { Garner Park. }\end{array}$ & $\begin{array}{l}\text { Frio R. at } \\
\text { Con Can. }\end{array}$ & $\begin{array}{l}\text { Prio R. at } \\
\text { Con Can. }\end{array}$ \\
\hline Date of collection & Aug. 28,1968 & Ju1y 29,1869 & Aug. 28,2968 & JuLy 29,1969 & Aug. 28,1968 & July 29,1969 & Aus. 28,3968 & July 20.1969 & Aug. 28.1968 & July 29,1969 \\
\hline Dlacharge (cfy)....... & 18.2 & 5.28 & 48.2 & 242 & 64.8 & a23 & 60.5 & 21.1 & 72.5 & 17.6 \\
\hline silice $\left(810_{2}\right) \ldots \ldots \ldots \ldots \ldots \ldots \ldots$ & -- & -- & - & -- & -- & - & $\cdots$ & -- & -- & $\cdots$ \\
\hline 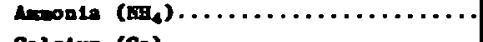 & .00 & .00 & .00 & -- & .00 & -- & .00 & .00 & .00 & .00 \\
\hline 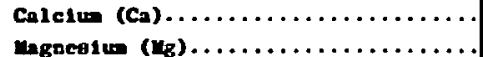 & $\overline{--}$ & -- & - & $\cdots$ & -- & $\cdots$ & -- & - & -- & -- \\
\hline & -- & $\begin{array}{l}-- \\
--\end{array}$ & $\begin{array}{l}-- \\
--\end{array}$ & -- & -- & $\because$ & - & - & -- & - \\
\hline Potassiu (x) $\ldots \ldots \ldots \ldots \ldots \ldots \ldots$ & -- & - & - & - & - & -- & - & -- & $\cdots$ & - \\
\hline Bicartonate $\left(\mathrm{H} \mathrm{O}_{2}\right) \ldots \ldots \ldots \ldots \ldots$ & 228 & -- & 258 & - & 250 & $\cdots$ & 231 & - & $216^{-6}$ & $204^{--}$ \\
\hline Carbonate $\left(\infty_{0}\right) \ldots \ldots \ldots \ldots \ldots \ldots$ & 0 &.- & 0 & -- & 0 & -- & 0 & - & o & o \\
\hline sulfate $\left(\mathrm{so}_{8}\right) \ldots \ldots \ldots \ldots \ldots \ldots$ & 7.4 & -. & 7.6 & -- & 7.6 & -. & 12 & -- & 13 & 16 \\
\hline Cnloride (C1) $\ldots \ldots \ldots \ldots \ldots \ldots \ldots$ & 12 & -- & 12 & -- & 14 & -- & 14 & -- & 15 & 16 \\
\hline 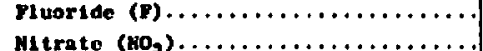 & 3.8 & $\ddot{.0}$ & $\overline{3}$ & $\ddot{-}$ & $\ddot{7}$ & $\because$ & 4. & $\ddot{8}$ & - & $\because$ \\
\hline & .00 & $\begin{array}{l}.0 \\
.00\end{array}$ & $\begin{array}{c}4.3 \\
.90\end{array}$ & $\overline{-}$ & $\begin{array}{l}3.7 \\
.00\end{array}$ & $\overline{--}$ & $\therefore \quad$. & 2.8 & $\begin{array}{l}5.0 \\
.00\end{array}$ & $\therefore \quad .80$ \\
\hline Phosphate $\left(P_{1}\right) \ldots \ldots \ldots \ldots \ldots \ldots \ldots$ & .02 & .03 & .04 & - & .01 & -- & .02 & .03 & .02 & .02 \\
\hline Boron (8) $\ldots \ldots \ldots \ldots \ldots \ldots \ldots \ldots \ldots \ldots$ & $\cdots$ & -- & -- & $\cdots$ & -- & -- & -- & -- & $\cdots$ & -- \\
\hline DLeselved sollds. & $\cdots$ & -- & -- & -- & -- & .. & -- & -- & -- & -- \\
\hline Hardaess $28 \mathrm{CaCO}_{2} \ldots \ldots \ldots$ & 201 & $=-$ & 243 & -- & 231 & -- & 216 & - & 206 & 148 \\
\hline $\begin{array}{l}\text { Noncarbonate hardness............. } \\
\text { Bodiua adsorption }\end{array}$ & 16 & -- & 32 & -- & 26 & $\cdots$ & 27 & - & 29 & $\mathbf{0}$ \\
\hline $\begin{array}{l}\text { Sodiuz adsorpt 1on } \\
\text { rat10 (BAB)............. }\end{array}$ & $=-$ & -- & -- & -- & -- & -- & -- & -- & -- & -- \\
\hline $\begin{array}{l}\text { Res1dunl sodiun carbonate (RSC) } \\
\text { (o1111equavalents por ifter).. }\end{array}$ & -- & -- & -- & $\cdots$ & -- & - & - & - & $\cdots$ & -- \\
\hline $\begin{array}{l}\text { 8pecific conduct ance } \\
\left.\text { (alcroabos at 23. } 25^{\circ}\right) . .\end{array}$ & 305 & -- & 147 & 444 & 457 & & 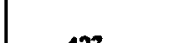 & 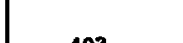 & $\because$ & \\
\hline pr. $\ldots \ldots \ldots \ldots \ldots \ldots \ldots$ & 7.6 & b7.6 & 7.6 & $\begin{array}{l}848 \\
67.5\end{array}$ & 020 & 848 & $\mathbf{4 2 7}$ & 103 & 414 & 362 \\
\hline Tesperature $\left({ }^{\circ} \mathrm{C}\right) \ldots \ldots \ldots$ & 25 & 26 & 24 & 26 & 7.7 & 07.3 & 7.8 & 67.7 & 7.7 & 7.9 \\
\hline Total collforn (per 100 al).... & 9700 & 2200 & 9200 & 3000 & 3600 & $\begin{array}{r}29 \\
0\end{array}$ & 1700 & $\begin{array}{r}29 \\
12000\end{array}$ & $\begin{array}{r}28 \\
6600\end{array}$ & $\begin{array}{r}30 \\
8200\end{array}$ \\
\hline Pocal colltore (per $100 \Rightarrow 1) \ldots$ & -- & - & -- & -- & -- & -- & -- & $\ldots$ & -- & - \\
\hline Fecal otreptococel (por 100 al). & -- & -- & - & -- & -- & $\cdots$ & -- & -- & -- & -- \\
\hline Blocbealcal oxygen deand (BOD). & .5 & .7 & .4 & -- &.$\theta$ & - & .8 & 1.1 & .5 & .7 \\
\hline Dotergents (1Bas) $\ldots \ldots \ldots \ldots \ldots$ & -- & -- & -- & -- & - & - & -- & -- & -- & -. \\
\hline Diseolved oxygen (DO)... & 7.3 & 9.2 & 7.9 & 8.8 & 9.0 & 11 & 9.4 & 8.9 & 8.9 & 8.7 \\
\hline Alualnew (A1)......... & -- & -- & $\cdots$ & -- & -- & $\cdots$ & -- & - & -- & -- \\
\hline Iron (Pe)............ & -- & -- & -- & - & -- & -- & -- & -- & -- & -- \\
\hline Manganese $\left(u_{n}\right) \ldots \ldots \ldots \ldots$ & -- & -- & - & -- & -- & -- & -- & $\cdots$ & - & -- \\
\hline Copper $(\mathrm{Cu}) \ldots \ldots \ldots \ldots \ldots$ & - & -- & -- & -- & -- & -- & -- & -- & -- & -- \\
\hline $\operatorname{zinc}(2 n) \ldots \ldots \ldots$ & -- & -- & - & -- & -- & - & -- & -- & -- & -- \\
\hline Lithlun (L1).............. & - & -- & -- & $\cdots$ & -- & -- & -- & -- & $\cdots$ & -- \\
\hline 8trostius (Sr)........ & -- & -- & -- & -- & -- & -- & -- & -- & -- & -- \\
\hline Mickol (bi1) $\ldots \ldots \ldots$. & $-\cdots$ & -- & -- & -- & -- & -- & -- & -- & -- & -- \\
\hline Lead $\left(P_{b}\right) \ldots \ldots \ldots \ldots \ldots \ldots$ & -- & $=-$ & - & - & -- & -- & -- & -- & -- & -- \\
\hline Broslde $(B r) \ldots \ldots \ldots \ldots \ldots \ldots$ & -- & -- & - & -- & -- & -- & -- & $-\bullet$ & - & -- \\
\hline Iodido (I) $\ldots \ldots \ldots \ldots \ldots \ldots$ & -- & - & -- & -- & -- & -- & -. & -- & -- & -- \\
\hline
\end{tabular}

Not8: No flow at alto 1 during both inveratsations. 
Tablo 2.--Qualley-of water data tron sites othor than wells and spriags in the San Antonio area--continued

(Bewults in allligrans per 11 ter except an indicated)

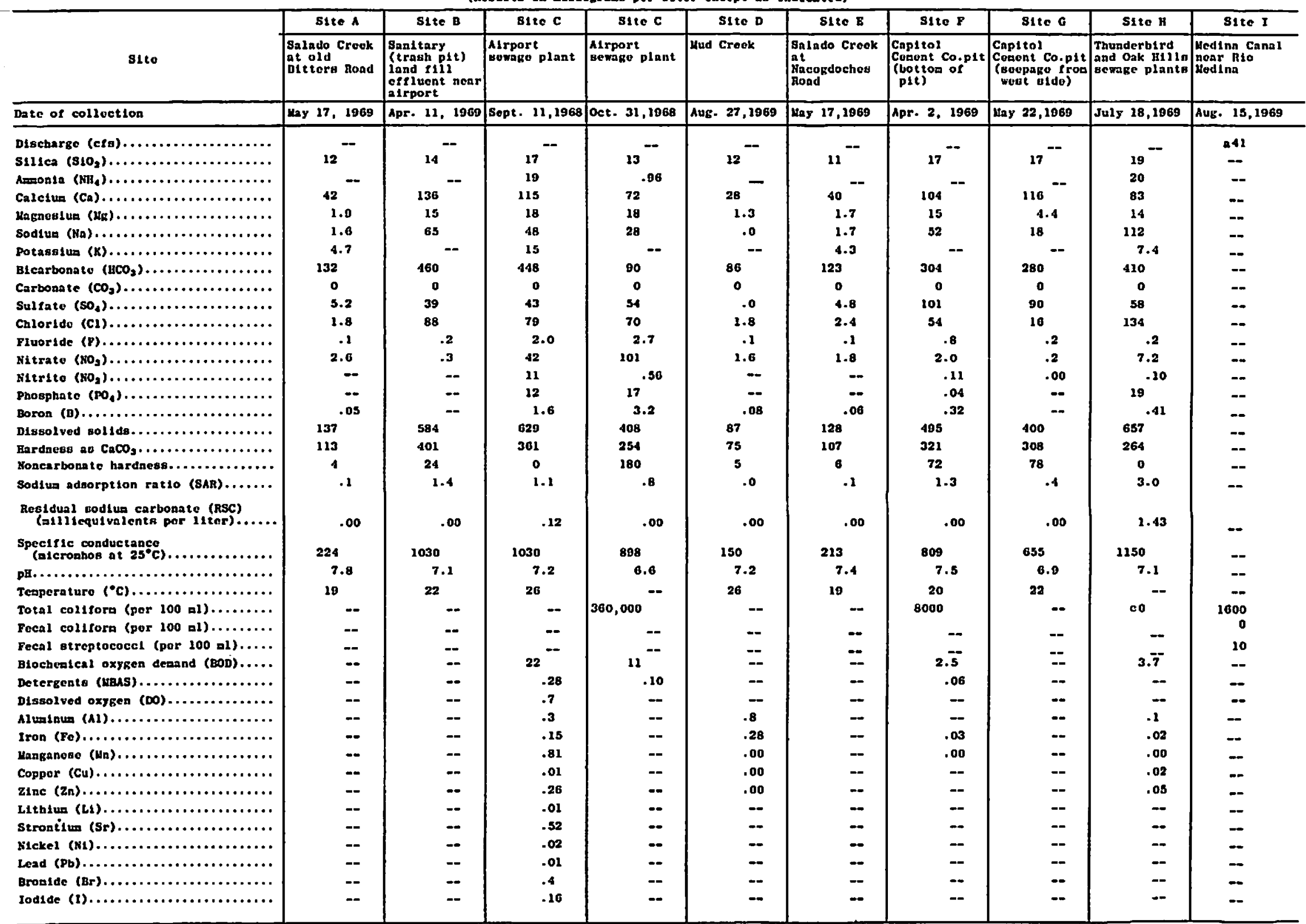

a Estizatod.

c ReBazplo of Aug. 25, 1969 contalned 7 colonles/100 al total collforn, o colonies/100 al tecal collfora, and 1400 colonleu/100 al focal atreptococel. 


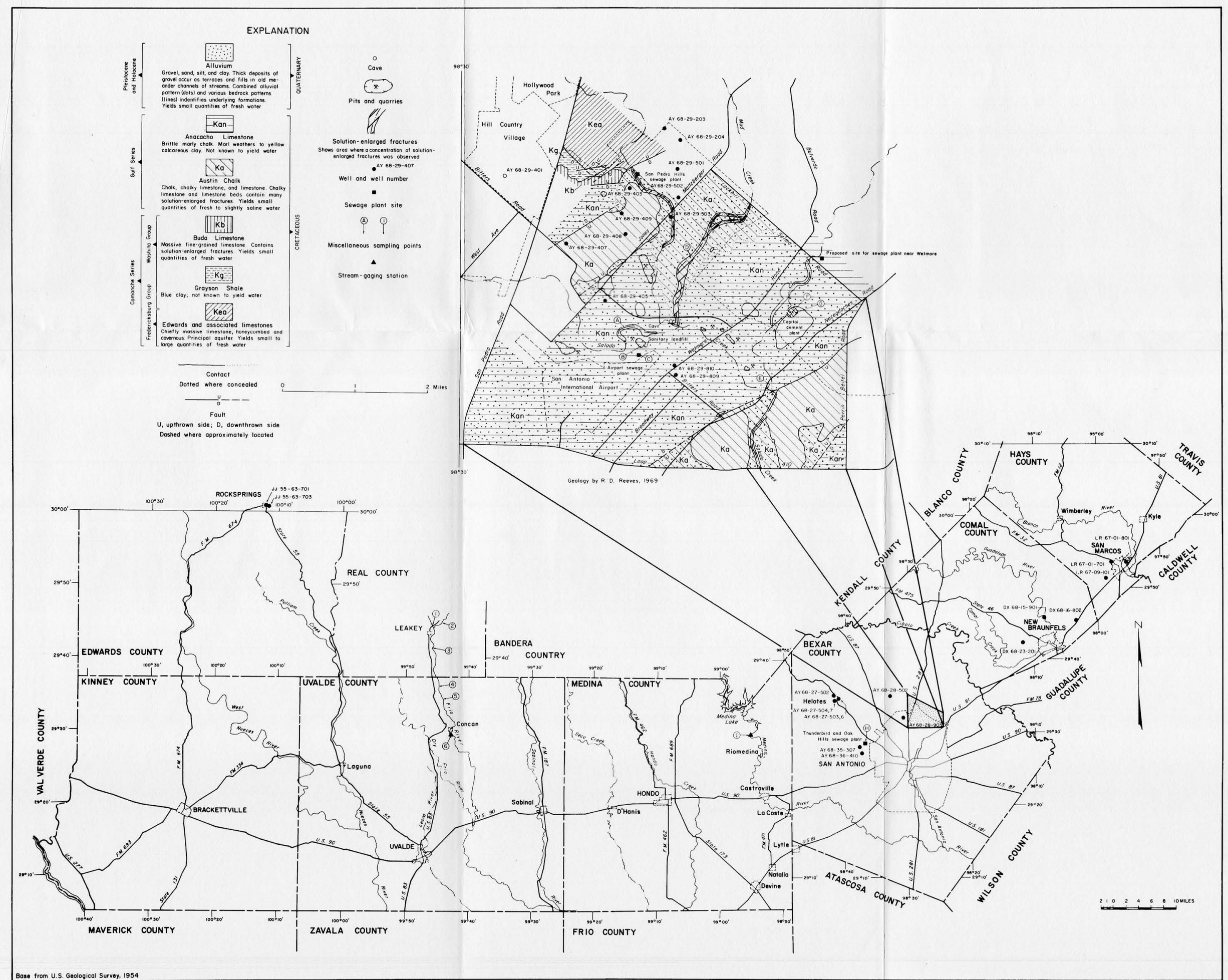

FIGURE I.- Water-quality data-collection sites in the San Antonio area and geology of part of north-central Bexar County 
Note: Large-format version of the original plate is on the following page. 




FIGURE I.-Water-quality data-collection sites in the San Antonio area and geology of part of north-central Bexar County 